\title{
Properties of Particle Production and System Evolution in BES- Wide Energy Range
}

\author{
Fu-Hu Liu ${ }^{D},{ }^{1}$ Sakina Fakhraddin, ${ }^{2,3}$ Raghunath Sahoo ${ }^{4},{ }^{4}$ \\ Edward K. Sarkisyan-Grinbaum, ${ }^{5,6}$ and Bhartendu K. Singh ${ }^{7}$ \\ ${ }^{1}$ Institute of Theoretical Physics, Shanxi University, Taiyuan, Shanxi 030006, China \\ ${ }^{2}$ Physics Department, College of Science \& Arts in RK, Qassim University, Buraidah, 51452 Qassim, Saudi Arabia \\ ${ }^{3}$ Physics Department, Faculty of Science, Sana'a University, P.O. Box 13499, Sana'a, Yemen \\ ${ }^{4}$ Discipline of Physics, School of Basic Sciences, Indian Institute of Technology Indore, Indore 453552, India \\ ${ }^{5}$ Experimental Physics Department, CERN, 1211 Geneva 23, Switzerland \\ ${ }^{6}$ Department of Physics, The University of Texas at Arlington, Arlington, TX 76019, USA \\ ${ }^{7}$ Physics Department, Banaras Hindu University, Varanasi 221005, India \\ Correspondence should be addressed to Fu-Hu Liu; fuhuliu@163.com
}

Received 28 May 2020; Accepted 29 May 2020; Published 4 June 2020

Copyright (c) $2020 \mathrm{Fu}-\mathrm{Hu}$ Liu et al. This is an open access article distributed under the Creative Commons Attribution License, which permits unrestricted use, distribution, and reproduction in any medium, provided the original work is properly cited.

The beam energy scan (BES) program at the Relativistic Heavy Ion Collider (RHIC) in Brookhaven and Super Proton Synchrotron (SPS) at CERN performs nucleus-nucleus collisions in the energy range of $3-39 \mathrm{GeV} /$ nucleon leading to high baryon density and temperature around the expected critical point of deconfinement phase transition. In addition, the Facility for Antiproton and Ion Research (FAIR) in GSI also performs nucleus-nucleus collisions in the BES energy region. The features of particle production and system evolution in these types of collisions attract high interest and are extensively studied both experimentally and theoretically. It is believed that above the critical energy of deconfinement phase transition, e.g., at RHIC and Large Hadron Collider (LHC), the properties of particle production and system evolution may differ from those at the BES (RHIC-BES, SPSBES, and FAIR). These properties are related to those of early universe considered to go through the stages of high baryon density and temperature.

Large amount of data obtained by high-energy experiments worldwide is being published to be scrutinized. Different signatures such as the chemical and kinetic freeze-out temperature, effective temperature, speed of sound, tension of string between valence quarks in colliding nucleons, minimum distance between these quarks, chemical potential of different types of particles and of different quark flavors, time evolution of collision system, and ratio of viscosity to entropy density are predicted by different models and can be obtained from the measured particle spectra allowing us to study the properties of the system formation and its evolution.

This special issue concerns many topics, for example, (i) description of particle distributions and correlations, studies of statistical laws and dynamical properties of particle production; (ii) studies of the properties of system evolution in beam energy scan program; (iii) extraction of different signatures based on the particle spectra, correlations, and yield ratios in BES-wide energy range; (iv) searches for the softest point of the equation of state and for the critical point of deconfinement phase transition; (v) comparison of the properties of particle production and system evolution at the BES energies with those at the RHIC and LHC energies; and (vi) comparison of the properties of particle production and system evolution in nucleus-nucleus collisions with those of small systems formed in particle collisions.

In the article "A Description of Transverse Momentum Distributions in $p+p$ Collisions at RHIC and LHC Energies" by J.-Q. Hui and Z.-J. Jiang, the authors assume the existence of longitudinal collective motion and long-range interactions in the hot and dense matter created in $p+p$ collisions. The 
relativistic hydrodynamics incorporating with the nonextensive statistics is used to analyze the transverse momentum distributions of the particles. The investigation of the present paper shows that the hybrid model can give a good description of the currently available experimental data obtained in $p+p$ collisions at RHIC and LHC energies, except for $p$ and $\bar{p}$ produced in the range of $p_{T}>3.0 \mathrm{GeV} / c$ at $\sqrt{s}=200 \mathrm{GeV}$. This article confirms that the hydrodynamics is suitable for the smaller colliding systems such as $p+p$ collisions.

In the article "Out-of-Equilibrium Transverse Momentum Spectra of Pions at LHC Energies" by A. N. Tawfik, the author investigates the transverse momentum spectra of positive pions measured in the ALICE experiment by utilizing two thermal approaches; one is based on degeneracy of nonperfect Bose-Einstein gas and the other imposes an ad hoc finite pion-chemical potential. It is shown that the inclusion of missing hadron states and the out-of-equilibrium contribute greatly to the characterization of pion production. A reproduction of these transverse momentum spectra is achieved at pion-chemical potential of $120 \mathrm{MeV}$, and this covers the entire range of transverse momentum. This article confirms a manifestation of not-yet-regarded anomalous pion production, which is related to "anomalous" protonto-pion ratios at top RHIC and LHC energies.

In the article "Contributions of Jets in Net Charge Fluctuations from the Beam Energy Scan at RHIC and LHC" by B. Ali et al., the authors study the dynamical net charge fluctuations in nucleus-nucleus collisions at RHIC-BES and LHC energies by a hadronic model. A Monte Carlo generator, HIJING, is used to generate events in two different modes, HIJING-default with jet quenching switched off and jet/minijet production switched off. A popular variable is used to study the net charge fluctuations in different centrality bins, and the findings are compared with the available experimental data. This article shows that nucleus-nucleus collisions can be treated as the superpositions of multiple nucleonnucleon collisions. The findings also reveal that the production of jets and minijets plays a dominant role in reducing the strength of particle correlations and fluctuations.

In the article "Centrality Dependence of Multiplicity Fluctuations in Ion-Ion Collisions from the Beam Energy Scan at FAIR" by A. Chandra et al., the authors investigate the multiplicity distributions and event-by-event fluctuations in Au-Au collisions at FAIR energies. Events corresponding to FAIR energies are simulated in the framework of UrQMD model. It is observed that the mean and the width of multiplicity distributions monotonically increase with beam energy. The trend of variations of dispersion with mean number of participating nucleons for the centrality-bin width of $5 \%$ is in accord with the Central Limit Theorem. The multiplicity distributions in various centrality bins are observed to obey KNO scaling. The trends of variations of scaled variance with beam energy are also found to support the KNO scaling predictions for larger collision centrality.

The article "Kinetic Freeze-Out Temperature and Transverse Flow Velocity in Au-Au Collisions at RHIC-BES Energies" by M. Waqas and B.-C. Li studies the midrapidity transverse momentum spectra of charged hadrons produced in central and peripheral $\mathrm{Au}-\mathrm{Au}$ collisions at RHIC-BES energies. The blast-wave model with the Boltzmann-Gibbs statistics is adopted to fit available experimental data measured by the STAR Collaboration at the RHIC-BES energies. The authors observe that the kinetic freeze-out temperature, transverse flow velocity, mean transverse momentum, and initial temperature increase with collision energy in the considered RHIC-BES energy range and with event centrality from peripheral to central collisions. This article confirms the complex correlation between kinetic freeze-out temperature and transverse flow velocity.

The article "Elliptic Flow of Identified Particles in $\mathrm{Pb}-\mathrm{Pb}$ Collisions at $\sqrt{s_{N N}}=5.02 \mathrm{TeV}^{\prime}$ " by E.-Q. Wang et al. investigates the elliptic flow coefficients of particles $\pi^{ \pm}, K^{ \pm}, p+\bar{p}$, $\Lambda+\bar{\Lambda}$, and $K_{S}^{0}$ produced in $\mathrm{Pb}-\mathrm{Pb}$ collisions at the centerof-mass energy of $\sqrt{s_{N N}}=5.02 \mathrm{TeV}$ by using a TsallisPareto-type function and the multisource thermal model. In the process of system evolution, deformation and translation occur for the isotropic emission source due to geometric structure, pressure gradient, and thermal diffusion effects, which leads to anisotropy in the azimuth distribution of identified particles. Based on these dynamic factors, the dependence of elliptic flow on transverse momentum is described. This article shows that source deformation plays a main role in the system evolution, and source translation can be neglected.

The article "Study of Spin-Spin Correlations between Quark and a Spin-1/2 Composite System" by S. Kaur and $H$. Dahiya studies the correlation between the fermion composite system and quark spins by using the light-cone quarkdiquark model. The authors do the calculations for $u$-quark and $d$-quark in the fermion system by considering different polarization configurations of both. The contribution from scalar and axial-vector diquarks is taken into account. The overlap representation of light-front wave-functions is used for the calculations. The spin-spin correlations for $u$ and $d$ quarks are presented in transverse impact-parameter plane and transverse momentum plane as well. This article also confirms that the spin-spin correlations are related to the Wigner distributions by integrating which over transverse momentum the measurable quantities can be extracted.

In the article "Transverse Momentum and Pseudorapidity Dependence of Particle Production in $\mathrm{Xe}-\mathrm{Xe}$ Collisions at $\sqrt{s_{N N}}=5.44 \mathrm{TeV}$ " by Z.-L. Guo et al., the Tsallis statistics is combined with a multisource thermal model through the collision-system configuration. The improved model is used to investigate the transverse momentum and pseudorapidity of charged particles produced in Xe-Xe collisions at $\sqrt{s_{N N}}=$ $5.44 \mathrm{TeV}$. The authors discuss detailedly the thermodynamic properties, which are taken from the transverse momentum distributions of charged particles for events with different centrality classes. The pseudorapidity spectra of charged particles for events with different centrality classes are also described consistently in the model. In addition, the model results estimate intuitively the longitudinal configuration of the collision system.

In the article "Investigation of Particle Distributions in $\mathrm{Xe}-\mathrm{Xe}$ Collision at $\sqrt{s_{N N}}=5.44 \mathrm{TeV}$ with the Tsallis Statistics" by H.-F. Zhao et al., the authors use the Tsallis statistics 
to investigate the transverse momentum distribution of charged particles produced in Xe-Xe collisions at the LHC. Meanwhile, the nuclear modification factor of the charged particles is studied. To obtain the related results, the authors use a new attempt which is improved by the Tsallis statistics in relaxation time approximation. Considering relaxation time approximation of the collision term, the authors achieve the final-state distribution by solving the Boltzmann transport equation, where the initial distribution is inserted consistently. The calculated results are approximately in agreement with the experimental data measured by the ALICE Collaboration.

In the article "Centrality Dependence of Multiplicity Fluctuations from a Hydrodynamical Approach" by H.-H. $\mathrm{Ma}$ et al., the authors investigate the centrality dependence of the multiplicity fluctuations regarding the recent measurements from STAR Collaboration. By employing a hydrodynamical approach, this article is dedicated to the noncritical aspects of the phenomenon. To be specific, in addition to the thermal fluctuations, finite volume corrections, and resonance decay at the freeze-out surface, this model is focused on the properties of the hydrodynamic expansion of the system and the event-by-event initial fluctuations. The real signal of the critical point can only be obtained after subtracting the background which is investigated in this article. The results are also compared to those of the hadronic resonance gas and transport models.

In the article "Study of Di-Muon Production Process in pp Collision in CMS Data from Symmetry Scaling Perspective" by S. Bhaduri et al., two methodologies, namely, MFDFA and MF-DXA analyses, are used for the study of scaling analysis of the dynamics of the di-muon production process using the primary data set of Run-A (2011) and Run-B (2012) of $p p$ collisions at 7 and $8 \mathrm{TeV}$ from CMS collaboration. The authors have analyzed how this scaling pattern has evolved from one rapidity range to the next one and how this change evolved from $7 \mathrm{TeV}$ to $8 \mathrm{TeV}$. Many interesting findings are obtained by the novel method of data analysis. This new approach has the potential to provide clue to possible different dynamics behind di-muon production (including beyond the standard model) in different rapidity domains which may change with higher and higher energy.

The article "Intermittency Study of Charged Particles Generated in $\mathrm{Pb}-\mathrm{Pb}$ Collisions at $\sqrt{s_{N N}}=2.76 \mathrm{TeV}$ Using EPOS3" by R. Gupta and S. K. Malik studies the charged particle multiplicity fluctuations in $\mathrm{Pb}-\mathrm{Pb}$ collisions for the events generated using EPOS3 (hydro and hydro+cascade). Normalized factorial moments $(F q)$ have been determined in the strict sense of intermittency being a power-law behavior of $F q$ with decreasing bin size. There is no significant scaling behavior in these moments with the decreasing bin size. The values of scaling exponent deduced for a few transverse momentum bins are greater than the value for the secondorder phase transition predicted by the Ginzburg-Landau theory. Fractal dimensions decrease with the order of the moment, which indicates the existence of a multifractal nature of the studied events.

This issue brings together a collection of articles. We hope this will be a useful issue for researchers working in related areas. Meanwhile, we regret that more manuscripts submitted for publication in this issue have not been accepted following the reviewer's reports.

\section{Conflicts of Interest}

The editors declare that they have no conflicts of interest regarding the publication of this special issue.

\section{Acknowledgments}

We sincerely thank all authors and reviewers of this special issue.

$\mathrm{Fu}-\mathrm{Hu} \mathrm{Liu}$

Sakina Fakhraddin

Raghunath Sahoo

Edward K. Sarkisyan-Grinbaum

Bhartendu K. Singh 